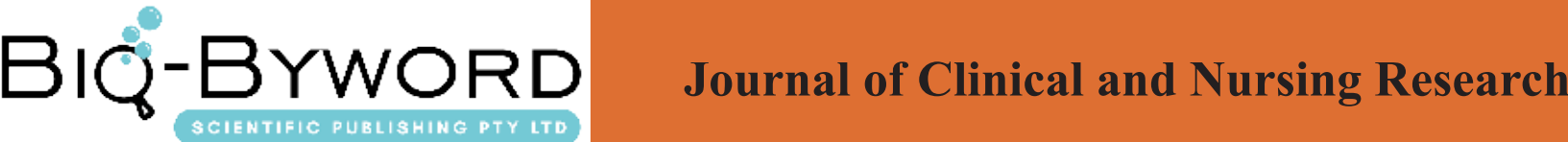

\section{Study on the clinical efficacy and mechanism of octreotide combined with thrombin in patients with liver}

\author{
Wang Meng, Zhang Xiuqin \\ Second People's Hospital of Shaanxi Province, Xi'an
}

\section{ARTICLE INFO}

Article history:

Published online: 15th July, 2017

Key words:

Octreotide

thrombin

cirrhosis

\section{ABSTRACT}

Objective: To observe the clinical efficacy of octreotide and thrombin in the treatment of patients with liver cirrhosis, and to explore the mechanism of octreotide and thrombin. Methods: 53 patients with cirrhosis were randomly divided into control group and observation group from January 2014 to January 2016. The control group of 26 patients, given octreotide treatment. Observation group of 27 cases, given octreotide and thrombin combination therapy. Comparison of two groups of patients with clinical efficacy and changes in liver function before and after treatment. Results: The effective rate (88.89\%) in the observation group was significantly higher than that in the control group (65.38\%), the difference was statistically significant $(\mathrm{p}<0.05)$. Before treatment, the difference of liver function between the two groups was not statistically significant $(p>0.05)$. After treatment, the liver function indexes of the two groups were improved, and the observation group was superior to the control group, the difference was statistically significant $(p<0.05)$. Conclusion: Octreotide can reduce the portal vein pressure, thrombin can improve the coagulation function of the body, the two combined treatment can significantly improve liver function in patients with liver cirrhosis, improve clinical efficacy, and thus help improve the quality of life of patients.

\section{Introduction}

Cirrhosis is a common clinical disease, clinical manifestations of jaundice, anemia, abdominal distension, ascites, etc. ${ }^{[1]}$. The disease incidence factors are more and irreversible and progressive development, which will endanger the safety of patients, increase the psychological pressure and mental burden of patients and 
reduce the quality of life of patients ${ }^{[2]}$. In order to effectively improve the clinical efficacy of patients with liver cirrhosis, this study randomly selected from January 2014 to January 2016 in our hospital treated 53 patients with cirrhosis as a study object to observe the combination of octreotide and thrombin in patients with cirrhosis clinical efficacy to explore the mechanism of action of octreotide and thrombin. Details are reported on below.

\section{Materials and methods}

\subsection{General information}

A total of 53 patients with cirrhosis treated in our hospital from January 2014 to January 2016 were randomly divided into control group and observation group. The average age $(53.47 \pm 5.26)$ years old; the duration of the course of 3 to 5 years, the average duration of disease $(3.21 \pm 1.02)$ years; the basic disease type ( 3 years), the average age (53.47 \pm 5.26$)$ : 10 cases of post-hepatitis cirrhosis, alcoholic cirrhosis in 6 cases, schistosomiasis cirrhosis in 5 cases, 5 cases of cholesteric cirrhosis. In the observation group, 27 cases were treated with octreotide and thrombin, and 15 males and 12 females were aged from 35 to 73 years (mean age $(53.52 \pm 5.24)$ years. The course of the course was 3 to 6 years, the average course of the disease $(3.18 \pm 1.12)$; Basic disease types: 11 cases of hepatitis cirrhosis, alcoholic cirrhosis in 5 cases, schistosomiasis cirrhosis in 6 cases, 5 cases of cholestatic cirrhosis. There was no significant difference in sex, age, duration of disease and underlying disease type between the two groups $(\mathrm{p}>0.05)$.

\subsection{Case selection criteria}

(1) Included in the standard: (1) The subjects are in line with the "cirrhosis of traditional Chinese and Western medicine diagnosis and treatment of consensus" ${ }^{[3]}$ on the diagnosis of cirrhosis of the liver: the clinical symptoms and signs of weight loss, mild fatigue, jaundice, anemia, Abdominal distension, ascites, etc.; B-show patients with liver shrinkage, abdominal cavity is liquid dark area, the liver surface was wavy or jagged, rugged, door splenic vein diameter was significantly widened, liver pods, echo Significantly enhanced and uneven. (2) This study has been approved by the Ethics Committee, and the patient signed informed consent.

(2) Exclusion criteria: (1) Chronic renal disease, cardiovascular and cerebrovascular diseases, autoimmune diseases. (2) Severe disturbance of consciousness, mental disorders, treatment compliance poor patients. (3)
Drug allergy history patients. (4) Lactation, pregnancy patients.

\subsection{Method}

All patients underwent conventional basal therapy such as fasting, bed rest, protein supplementation, gastric mucosal protection, and nutritional support.

1.3.1 Observation group given octreotide and thrombin combination therapy

Intravenous infusion of octreotide (manufacturer: Chengdu Li Site Pharmaceutical Co., Ltd., specifications: $1 \mathrm{ml}$ : $0.1 \mathrm{mg}$, Zhunzi H20051570) $0.1 \mathrm{mg}$, 5\% glucose injection (Manufacturer: Chongqing Pharmaceutical Pharmaceutical Co., Ltd., specifications: $250 \mathrm{ml}$ : $12.5 \mathrm{~g}$, Chinese medicine word H50020628) 500mL, 1 day, continuous treatment for 6 days. (Manufacturer: Shaanxi Meichen Pharmaceutical Co., Ltd., specifications: $500 \mathrm{ml}: 4.5 \%$ ), the amount of sodium chloride injection (manufacturer: Shaanxi Meichen Pharmaceutical Co., Ltd., specifications: 500ml: 4.5 G, Chinese medicine Zhunzi H61021966) 15mL, diluted evenly, the tube injection or oral, $6 \mathrm{~h}$ once, continuous treatment for 6 days.

\subsubsection{Control group given octreotide treatment}

Given octreotide treatment, treatment same with the observation group with octreotide treatment, and one day once for continuous treatment for 6 days.

\subsection{Observe the indicators and assessment criteria}

After 6 consecutive days of treatment (1) Compared the clinical efficacy of two groups of patients. Evaluation criteria: (1) Remarkably effective: abdominal wall, jaundice, bloating, ascites and other clinical signs and symptoms, spleen increased, hepatobiliary narrow, portal vein was significantly widened and other imaging findings disappeared or improved, significantly improved liver function. (2) Effective: clinical signs, symptoms and imaging performance improved, improved liver function. (3) Invalid: clinical signs, symptoms and imaging performance, liver function is not improved or even increased. Treatment efficiency $=($ marked + effective) / total number of cases $\times 100 \%$. (Total Bilirubin, TBIL), Albumin, Alb, Glutamic Oxaloacetic Transaminase (AST) were measured before and after treatment.

\subsection{Statistical methods}

This study was used to analyze the data SPSS18.0 for Windows data, 2 groups of patients with clinical efficacy (\%), using $\chi^{2}$ test. 2 groups of patients before 
Table 1 Comparison of two groups of patients with clinical efficacy (n,\%)

\begin{tabular}{|c|c|c|c|c|c|}
\hline Group & Cases & Remarkably effective & Effective & Invalid & Treatment efficacy \\
\hline Control group & 26 & 8 & 9 & 9 & $17(65.38)$ \\
\hline $\begin{array}{c}\text { Observation } \\
\text { group }\end{array}$ & 27 & 14 & 10 & 3 & $24(88.89)^{*}$ \\
\hline$\chi^{2}$ & - & - & - & - & 4.1777 \\
\hline$p$ & - & - & - & - & 0.0410 \\
\hline
\end{tabular}

Note: * compared with the control group, $\mathrm{p}<0.05$.

and after treatment changes in liver function $(\overline{\mathrm{x}} \pm \mathrm{s})$ said, using $\mathrm{t}$ test. The difference was statistically significant with $\mathrm{p}<0.05$.

\section{Results}

2.1 Comparison of two groups of patients with clinical efficacy

The effective rate of treatment group (88.89\%) was significantly higher than that of the control group $(65.38 \%)$, the difference was statistically significant ( $p$ $<0.05)$.

2.2 Comparison of two groups of patients before and after treatment of liver function changes

Before treatment, the difference of liver function between the two groups was not statistically significant $(\mathrm{p}>0.05)$. After treatment, the liver function indexes of the two groups were improved, and the observation group was superior to the control group, the difference was statistically significant $\mathrm{P}<0.05$ ), see Table 2 .

\section{Discussion}

Cirrhosis is caused by a variety of causes of nodular regeneration of the body, extensive liver cell degeneration and necrosis, hepatic diffuse fibrosis and false lobular formation and liver lobular structure of chronic diseases ${ }^{[4]}$. The disease causes more, such as alcoholism, biliary tract disease, autoimmune injury, chronic viral hepatitis, schistosomiasis infection, including cirrhosis in patients with chronic viral hepatitis as the main cause ${ }^{[5]}$. Liver cirrhosis is divided into the compensatory and decompensatory stage, liver cirrhosis decompensated patients with liver function test no obvious abnormalities, but the liver histopathological changes are more obvious, generally by adjusting the diet and living habits to avoid being emotional, overwork, assisted clinical drug therapy, etc. It can delay the progression of the disease, promote disease rehabilitation. However, due to cirrhosis of the compensatory phase of patients with clinical symptoms and signs are not obvious, not specific, leading to clinical treatment of patients with the disease has developed into decompensated liver cirrhosis, the main clinical manifestations of portal hypertension, hepatocellular dysfunction. Often complicated by hepatic encephalopathy, gastrointesti-

Table 22 groups of patients before and after treatment of liver function changes $(\overline{\mathrm{x}} \pm \mathrm{s})$

\begin{tabular}{|c|c|c|c|c|c|c|c|}
\hline \multirow{2}{*}{ Group } & \multirow{2}{*}{ Cas- } & \multicolumn{2}{|c|}{ TBIL $(\mu \mathrm{mol} / \mathrm{L})$} & \multicolumn{2}{c|}{ ALT $(\mathrm{U} / \mathrm{L})$} & \multicolumn{2}{c|}{ AST (U/L) } \\
\cline { 3 - 8 } & Before treat- & $\begin{array}{c}\text { After treat- } \\
\text { ment }\end{array}$ & Before treatment & $\begin{array}{c}\text { After treat- } \\
\text { ment }\end{array}$ & Before treatment & After treatment \\
\hline $\begin{array}{c}\text { Control } \\
\text { group }\end{array}$ & 26 & $46.32 \pm 16.87$ & $30.52 \pm 11.31$ & $262.47 \pm 133.39$ & $67.32 \pm 19.21$ & $182.36 \pm 103.57$ & $59.32 \pm 18.35$ \\
\hline $\begin{array}{c}\text { Observation } \\
\text { group }\end{array}$ & 27 & $46.41 \pm 16.92$ & $24.29 \pm 8.68^{*}$ & $259.63 \pm 129.75$ & $55.64 \pm 17.98^{*}$ & $181.25 \pm 104.54$ & $47.78 \pm 17.96^{*}$ \\
\hline $\mathrm{t}$ & - & 0.0194 & 2.2548 & 0.0786 & 2.2862 & 0.0388 & 2.3137 \\
\hline $\mathrm{p}$ & - & 0.9846 & 0.0285 & 0.9377 & 0.0264 & 0.9692 & 0.0248 \\
\hline
\end{tabular}

Note: * compared with the control group, $\mathrm{p}<0.05$. 
nal bleeding, liver and kidney syndrome, the patient's physical health and normal life, learning a greater threat [6]. Therefore, the clinical should actively explore effective treatment to improve the quality of life of patients.

The study group was given octreotide treatment, the observation group gave octreotide and thrombin combination therapy,

The results showed that the effective rate $(88.89 \%)$ in the observation group was significantly higher than that in the control group $(65.38 \%)$, the difference was statistically significant $(p<0.05)$. Indicating that octreotide and thrombin combination therapy can significantly improve the clinical efficacy of patients with liver cirrhosis. Octreotide is a synthetic drug, the role of similar to the natural endogenous somatostatin, and half-life longer than the natural endogenous somatostatin 30 times, with strong and long time and other characteristics ${ }^{[7]}$. Study [8] found that octreotide into the body can effectively inhibit the digestive system secretion of digestive juice, which can inhibit gastrointestinal motility, to promote the body off the blood flow, portal pressure decreased. Thrombin is a sterile preparation, extracted from pig blood or bovine blood, serine protease, can promote the fibrinogen to fibrin conversion, has a good coagulation, anticoagulant properties ${ }^{[9]}$. Cirrhosis patients with gastrointestinal bleeding and other clinical symptoms, if the clinical treatment of patients with cirrhosis, only given octreotide treatment, it will cause the body portal pressure decreased, hemostatic effect is poor. Thrombin can activate the body platelets, to ensure the stability of blood clots. Combination therapy of the two drugs can not only improve the clinical symptoms of patients with cirrhosis, but also to promote the patient's own activity increased, which can significantly improve the clinical efficacy of patients with liver cirrhosis ${ }^{[10]}$. The results of this study showed that the difference of liver function between the two groups was not statistically significant $(\mathrm{p}>0.05)$. After treatment, the liver function indexes of the two groups were improved, and the observation group was better than the control group. $(\mathrm{P}<0.05)$, indicating that combination therapy with octreotide and thrombin can significantly improve liver function in patients with liver cirrhosis, which will help improve the quality of life of patients. Octreotide not only has thyroid stimulating hormone, growth hormone and other pathological secretion of inhibition, but also inhibit the inhibition of trypsin, gastric acid, insulin, glucagon secretion, direct protection of the body pancreatic parenchymal membrane effect. Cirrhosis patients with portal pressure increased significantly, liver damage, the application of octreotide can promote the body's portal pressure and inflammatory mediators decreased, reduce the body's gastrointestinal blood flow, reduce liver damage and gastric esophageal variceal tension, which can be effectively improved patients with liver function ${ }^{[11]}$. Liver is the synthesis of thrombin in the body, the clinical evaluation of liver function is an important indicator of coagulation function, cirrhosis patients will cause serious complications of thrombin synthesis, reduce prothrombin activity, induced bleeding. After the application of thrombin in patients with cirrhosis, the surface of the body blood in a short period of time to form a more stable clot, can effectively reduce the body bleeding, can be used as tissue grafts, skin fixatives, adhesives, combined with octreotide treatment, thus can significantly improve liver function in patients with liver cirrhosis, which contribute to a significant increase in the quality of life of patients ${ }^{[12]}$.

In combination, octreotide combined with thrombin can significantly improve the clinical efficacy of patients with liver cirrhosis, improve liver function, and thus contribute to the improvement of quality of life of patients with important clinical promotion and application value.

\section{References}

[1] Zhang Chao. Thrombin Combined With Octreotide in the Treatment of Cirrhosis with Upper Gastrointestinal Bleeding $[\mathrm{J}]$. Modern digestion and interventional therapy, 2015, 20 (5): 526-527.

[2] Li Xiaohong. Octreotide Combined With Thrombin In The Treatment Of Liver Cirrhosis With Upper Gastrointestinal Bleeding Effect [J]. Journal of Clinical Pharmacology, 2013, 6 (19): 66.

[3] Liu Chenghai, Wei Beihai, Yao Shukun. Cirrhosis of traditional Chinese and Western Medicine Diagnosis and Treatment of Consensus [J]. Chinese Journal of Integrated Traditional and Western Medicine, 2011, 19 (04): 277-279.

[4] Deng Shukai. Octreotide Combined With Thrombin in the Treatment of Cirrhosis with Upper Gastrointestinal Bleeding Clinical Efficacy Observation [J]. Practical Journal of Clinical Medicine, 2015, 19 (13): 111-112.

[5] Lin Shuhong, Chen Haijun, Tang Ying, et al. Octreotide Combined With Thrombin in the Treatment of Cirrhosis with Esophageal Variceal Bleeding Effect of Observation [J]. Modern digestion and interventional therapy, 2015, 20 (3): 297-298. 
[6] Li Haiqin. Octreotide Combined With Thrombin in the Treatment of Cirrhosis with Upper Gastrointestinal Bleeding [J]. Chinese Journal of Practical Medicine, 2014, 41 (21): 91-92.

[7]Zhang, Y.,Liu, Z.,Liu, X. et al. Prediction of Octreotide Efficacy By Electrogastrography in the Treatment of Patients with Esophageal Variceal Hemorrhage[J]. Physiological measurement,2013,34(7):799-812.

[8] Liu Jianhua, Zhu Fenfang. Octreotide Combined With Thrombin In The Treatment Of Cirrhosis With Upper Gastrointestinal Bleeding Effect [J]. Bethune Medical Journal, 2014, 12 (1): 21-23.

[9] Li Yongjiang. Thrombin Combined with Octreotide in the Treatment of Cirrhosis with Upper Gastrointestinal Bleeding Clinical Analysis [J]. Modern Diagnosis and Treatment, 2015, 26 (18): 4169-4170.

[10] Shao Hongzheng, Hu Zhiyong, Liu Rong et al .12 Clinical Manifestations Of 12 Cases Of Cirrhosis With Non-Variceal Upper Gastrointestinal Bleeding [J]. Hubei, 2015,20 (3): 226-228.

[11] Kim, Seon Young, Kim, Ji-Eun, Kim, Yoon Jun et al. Prominent Protein Z-Induced Thrombin Inhibition In Cirrhosis: A New Functional Assay For Hypercoagulability Assessment [J]. Journal of gastroenterology and hepatology, 2015, 30 (4): 784-793.

[12] Cao Wei. Acidized Intestinal Treatment of Cirrhosis with Gastrointestinal Dysfunction in Patients with Blood Ammonia Levels and Significance [J]. Liver, 2015, 20 (6): 466-468. 